\title{
Algoritmos de derivación y confirmación diagnóstica de citología cervical atípica: desafíos para la actualización
}

\author{
Fanny López-Alegría, PhD ${ }^{1 a}$, Estela Arcos G., MSc ${ }^{1 a}$, Luis González E. MSc ${ }^{1 b}$, \\ Dino Roberto Soares De Lorenzi 2 , Orlando Quezada P. $3 \mathrm{C}$
}

${ }^{1}$ Facultad de Enfermería, Universidad Andres Bello, Santiago, Chile. ${ }^{2}$ Departamento de Tocoginecología, Universidade de Caxias do Sul, Brasil. ${ }^{3}$ Laboratorio de Citología del Servicio de Anatomía Patológica del Complejo Asistencial Barros Luco, Santiago, Chile.

a Matrona. ${ }^{b}$ Enfermero. ${ }^{c}$ Tecnólogo Médico.

\section{RESUMEN}

El Programa Nacional de Pesquisa y Control del Cáncer Cervicouterino de Chile ha contribuido al descenso sostenido de la mortalidad por cáncer de cuello uterino. Para la reducción de esta mortalidad ha sido fundamental la citología exfoliativa del cérvix, la que no está exenta de resultados inciertos. En este sentido, los frotis clasificados como atípicos se consideran ambiguos por la presencia de anomalías celulares de difícil determinación, lo que se traduce en un diagnóstico de probabilidad incierta. En la literatura nacional como internacional, se manifiesta un notorio interés por unificar la nomenclatura citológica cervical y los algoritmos de derivación y confirmación diagnóstica, para el manejo clínico de las mujeres con anomalías citológicas cervicales y lesiones precursoras de cáncer cervicouterino. Por lo anterior, se considera relevante los estudios que proporcionen evidencia clínica epidemiológica actualizada, que permitan optimizar el cumplimiento del Programa Nacional de Cáncer Cervicouterino, conducentes al logro de los Objetivos Sanitarios del período 2011-2020.

\section{PALABRAS CLAVE: Papanicolaou, frotis atípico, células escamosas atípicas, algoritmo}

\section{SUMMARY}

The National Research and Control of Cervical Cancer in Chile had contributed to the sustained decline in mortality from cervical cancer. The exfoliative cytology of the cervix has been to reduce this mortality, which is not without uncertain results. The smears classified as atypical are considered ambiguous by the presence of cellular abnormalities difficult to determine, resulting in a diagnosis of uncertain probability. The literature, both nationally and internationally, is widespread interest to unify the nomenclature cervical cytology and the support of the derivation algorithms for the clinical management of women with cytologic abnormalities cervical and cervical cancer precursor lesions. Therefore, it is relevant to perform studies the provide updated epidemiological clinical evidence, to optimize the performance of the National Program for the achievement of health objectives for the period 2011-2020.

KEY WORDS: Pap test, atypical smear, atypical squamous cells, algorithm 


\section{INTRODUCCIÓN}

En los Objetivos de Desarrollo del Milenio (ODM), no se menciona como estrategia específica la prevención del cáncer cervicouterino, sin embargo, implícitamente se valora la contribución que aportan estas acciones de salud al logro de los ODM (1). En este contexto, el cáncer cervicouterino ha sido definido como "un caso de estudio para equidad en salud" (1), dado que el $85 \%$ de las muertes por esta causa, ocurren en los países en desarrollo, donde los programas de pesquisa no han tenido la efectividad esperada (1).

En Chile, el Programa Nacional de Pesquisa y Control del Cáncer Cervicouterino ha contribuido al descenso sostenido de la mortalidad por cáncer de cuello uterino, modificando la tasa de 11,9 por 100.000 mujeres en el año 1990 a 7,5 por 100.000 mujeres en 2008 (2). El objetivo primordial de este programa es "disminuir la tasa de mortalidad e incidencia por cáncer invasor a través de la detección en etapas preinvasoras y tratamientos adecuados y oportunos" (3).

Para el logro de este propósito, ha sido fundamental el aporte de la aplicación del test de tamizaje de citología exfoliativa del cérvix, que desde sus primeras descripciones morfológicas realizadas por George Papanicolaou, se ha mantenido sin cambios significativos. Lo que ha variado es la terminología para informar estas descripciones, especialmente la comunicación de resultados inciertos, como son las atipias citológicas (4).

\section{ANTECEDENTES DE LA NOMENCLATURA DE LA CITOLOGÍA CERVICAL ATÍPICA}

En 1943, Papanicolaou ideó un sistema de clasificación numérica para los hallazgos de los informes de la citología cervical, dividiendo las células exfoliadas en cinco clases, siendo la Clase II definida como "atípicas, pero sin evidencia de malignidad". A fines de la década del 80 , se incrementó los resultados clasificados como "los mal definidos términos de atipias, para designar anomalías o lesiones menos graves que displasias". Incluso en aquella época, estos resultados inciertos representaban una dificultad para el manejo clínico de la paciente $(5,6)$.

Para hacer frente a esta percepción de "caos de diagnóstico" en la citología cervical, el Instituto Nacional del Cáncer de Estados Unidos patrocinó, en diciembre de 1988, un Workshop con el fin de adoptar una terminología uniforme para denominar los resultados de la citología cervical. Esto originó una nueva clasificación, el Sistema Bethesda. En esta, los expertos definieron el término de "células atípicas" como los casos en que los hallazgos citológicos son de naturaleza indeterminada, creándose las categorías "células escamosas atípicas de significado indeterminado" y "células glandulares atípicas de significado indeterminado". Los informes citológicos, en los cuales las células eran descritas como atípicas, debían incluir una recomendación para su posterior evaluación con el fin de ayudar al clínico (7).

Tres años después (1991), el Instituto Nacional del Cáncer patrocinó un segundo Workshop para revisar el Sistema Bethesda. En este, se propuso que el citopatólogo valorara si los hallazgos de atipia escamosa o glandular de significado indeterminado favorecían un proceso reactivo o premaligno/ maligno, con el fin de facilitar el manejo de la paciente $(8,9,10)$.

Para actualizar nuevamente la nomenclatura del Sistema Bethesda, se realizó un tercer Workshop en abril del 2001, el que rige actualmente en los informes de la citología cervical (11). Una de las modificaciones más importantes se realizó en la categoría "células escamosas atípicas de significado indeterminado" que ahora se denomina "células escamosas atípicas (ASC)" y se subdividen en "células escamosas atípicas de significado indeterminado (ASCUS)" y "células escamosas atípicas no excluyente de lesión escamosa de alto grado (ASC-H)". Estas últimas fueron definidas con el fin de distinguir una lesión con posibilidades de acercarse a carcinoma invasor de células escamosas, que tiene una conducta clínica diferente $(12,13)$.

\section{EXPERIENCIA INTERNACIONAL SOBRE DI- RECTRICES EN PRÁCTICAS CLÍNICAS DE LA CITOLOGÍA CERVICAL}

Con la intención de definir conductas clínicas en relación a las anormalidades cervicales leves para apoyar la formulación de directrices clínicas, el Instituto Nacional del Cáncer de Estados Unidos, entre los años 1996-2000, patrocinó el mayor de los ensayos clínicos realizados hasta hoy en relación a este tema, el ASCUS-LSIL Triage Study (ALTS) (14). En este ensayo clínico se escogieron al azar y distribuyeron equitativamente 3.488 mujeres con frotis ASCUS en 3 protocolos de conductas clínicas: i) colposcopia inmediata, ii) pruebas de ADN para detección del Virus del Papiloma Humano (VPH) y iii) seguimiento con citología. En estos protocolos se realizó un seguimiento por 24 meses a través de histología, que demostró que la tasa acumulativa de neoplasia cervical intraepitelial grado 3 (NIC 3) fue similar en los 3 protocolos, es decir, $8,3 \%$ para 
colposcopia, 8,7\% en VPH y 9,3\% para citología $(15,16,17)$. En el caso de las otras conductas de células escamosas atípicas no excluyentes de lesión de alto grado (ASC-H), estas fueron basadas en un consenso de expertos (18).

Paralelo al ensayo clínico ALTS, en el 2000 el Colegio Americano de Patólogos realizó una encuesta (19). Esta demostró que la mayoría de los laboratorios proporcionan recomendaciones de seguimiento en los informes de ASCUS, siendo la más frecuente repetir el test de Papanicolaou en diferentes intervalos de tiempo y la menos recomendada, la colposcopia con biopsia.

La gran diversidad de resultados citológicos con diagnóstico de atipias, creó controversias sobre su manejo clínico, lo que reforzó la intención de generar un consenso en las conductas clínicas. Esta idea se materializó en las primeras directrices prácticas clínicas para mujeres con anormalidad citológica cervical y lesiones precursoras de cáncer cervical implementadas en Estados Unidos, basadas en evidencias (20). Estas directrices fueron copatrocinado por la Sociedad Americana de Patología Cervical y Colposcopia (ASCCP) realizada en septiembre de 2001(21). En estas, para el caso de ASCUS, no se llegó a un consenso de conducta única, recomendando adoptar una de las tres siguientes: i) la realización de dos test de Papanicolaou a los 6 y 12 meses; ii) colposcopia inmediata y iii) pruebas de ADN para los tipos de alto riesgo del Virus del Papiloma Humano (VPH). En los casos de informes citológicos con células escamosas atípicas que no excluye una lesión intraepitelial de alto grado (ASCH) y las atipias glandulares (AGC), se recomendó la colposcopia inmediata como estrategia única y definida, debido a la alta probabilidad de lesión de alto grado $(21,22)$.

Después de la implementación de estas directrices clínicas, se realizaron investigaciones bajo los parámetros contenidos en ellas, cuyos resultados hicieron evidente la necesidad de realizar algunos cambios, específicamente, en el manejo de las poblaciones de mujeres adolescentes, embarazadas y posmenopáusicas (23).

Como consecuencia de lo anterior, en la segunda conferencia patrocinada por la ASCCP en 2006, se elaboraron nuevas directrices específicas para estos grupos de mujeres, en la cual se confirmaron las tres conductas clínicas de ASCUS con variación de los intervalos de tiempo para su aplicación (repetición de Papanicolaou, la colposcopía inmediata y la prueba de VPH de alto riesgo). Para los ASC-H se ratificó la colposcopia inmediata. En una evaluación inicial, para las atipias glandulares (AGC), que son poco frecuentes y habitualmente asociadas a una significativa condición subyacente neoplásicas, se propuso colposcopia con legrado endometrial y endocervical para todas las subcategorías de AGC $(23,24,25)$.

\section{INVESTIGACIONES BAJO LA NOMENCLATURA DEL SISTEMA BETHESDA 2001}

La uniformidad de criterio tanto en la nomenclatura de la citología cervical atípica, como en las directrices de conducta clínica para su confirmación diagnóstica, han permitido realizar estudios comparativos de prevalencia y seguimiento longitudinal para conocer la historia natural de la citología cervical atípica. Los estudios realizados muestran que la prevalencia de frotis ASCUS varía de $0,2 \%$ en Mauritania a 7,8\% en Egipto (26-34). En América Latina, específicamente en Brasil, encontramos diferencias de entre 0,32 a 3,96\% (35-37). En Chile, en el área Sur de la Región Metropolitana de Santiago, se encontró una prevalencia de $0,67 \%$ en los Papanicolaou realizados el año 2005 (38). Con anterioridad, el Instituto Nacional de Cáncer de los Estados Unidos (1992), definió para el diagnóstico de ASCUS una prevalencia esperada del $5 \%$ de las citologías cervicales y que una frecuencia mayor puede estar representando una clasificación citológica inadecuada $(6,8)$. En relación al ASC-H, la prevalencia varió de $0,22 \%$ en la India hasta $8,8 \%$ en Sudáfrica, entre el 2004 a 2006 (27,39-45). Esta última investigación se diferencia de la gran mayoría de los estudios, en los cuales la prevalencia es inferior a $1 \%$ (44).

Respecto al seguimiento cito-histológico de la historia natural de las atipias, se han realizado varios estudios que reportan un diagnóstico definitivo de lesiones de alto grado entre $0,7-16 \%$ y para carcinomas invasores de $0-1,4 \%(33,37,45-47)$. En Chile, en un estudio de seguimiento cito-histológico del ASCUS en 154 mujeres, se informó que 21,4\% de las citologías atípicas evolucionaron a lesiones de alto grado y un $1,3 \%$ a carcinomas invasores (38). Por lo anterior, es importante realizar seguimiento cito-histológico a las mujeres con frotis atipicos, porque un porcentaje evolucionan a lesiones de alto grado, lo que constituye el punto crítico para tomar decisiones de conducta clínica. A diferencia de los estudios anteriores, existen investigaciones que parten de la patología, como el de Pedrosa y cols (2003), quienes realizaron un estudio retrospectivo, en el cual concluyeron que aproximadamente $2 / 3$ de los casos histológicos de neoplasia intraepitelial cervical (NIC $2+$ ) fueron precedidos por anormalidades citológicas menores (6). 
En relación a los estudios de ASC-H, la prevalencia de lesiones NIC 2+ son definitivamente superiores, desde $22,5 \%$ encontrado en el seguimiento de 89 mujeres postmenopáusicas en Memphis, Estados Unidos, hasta un $79,9 \%$ en el seguimiento cito-histológico de 517 mujeres en Alberta, Canadá $(41,42)$.

\section{EXPERIENCIA EN CHILE EN LA FORMULACIÓN DE GUÍAS PRÁCTICAS CLÍNICAS}

El Ministerio de Salud de Chile implementó en
2006 la Guía Clínica Cáncer Cervicouterino para apoyar las decisiones clínicas de los profesionales de salud. Estas guías, basadas en las primeras directrices de consenso de Wright (2002), contienen el algoritmo de derivación para el manejo clínico de mujeres con anomalías citológicas cervicales y lesiones precursoras de cáncer cervicouterino (Figura 1) (48). Además, se complementa el algoritmo de confirmación diagnóstica, que indica los tipos de exámenes y procedimientos a realizar en las anomalías citológicas cervicales y lesiones precursoras de cáncer cervicouterino (Figura 2).

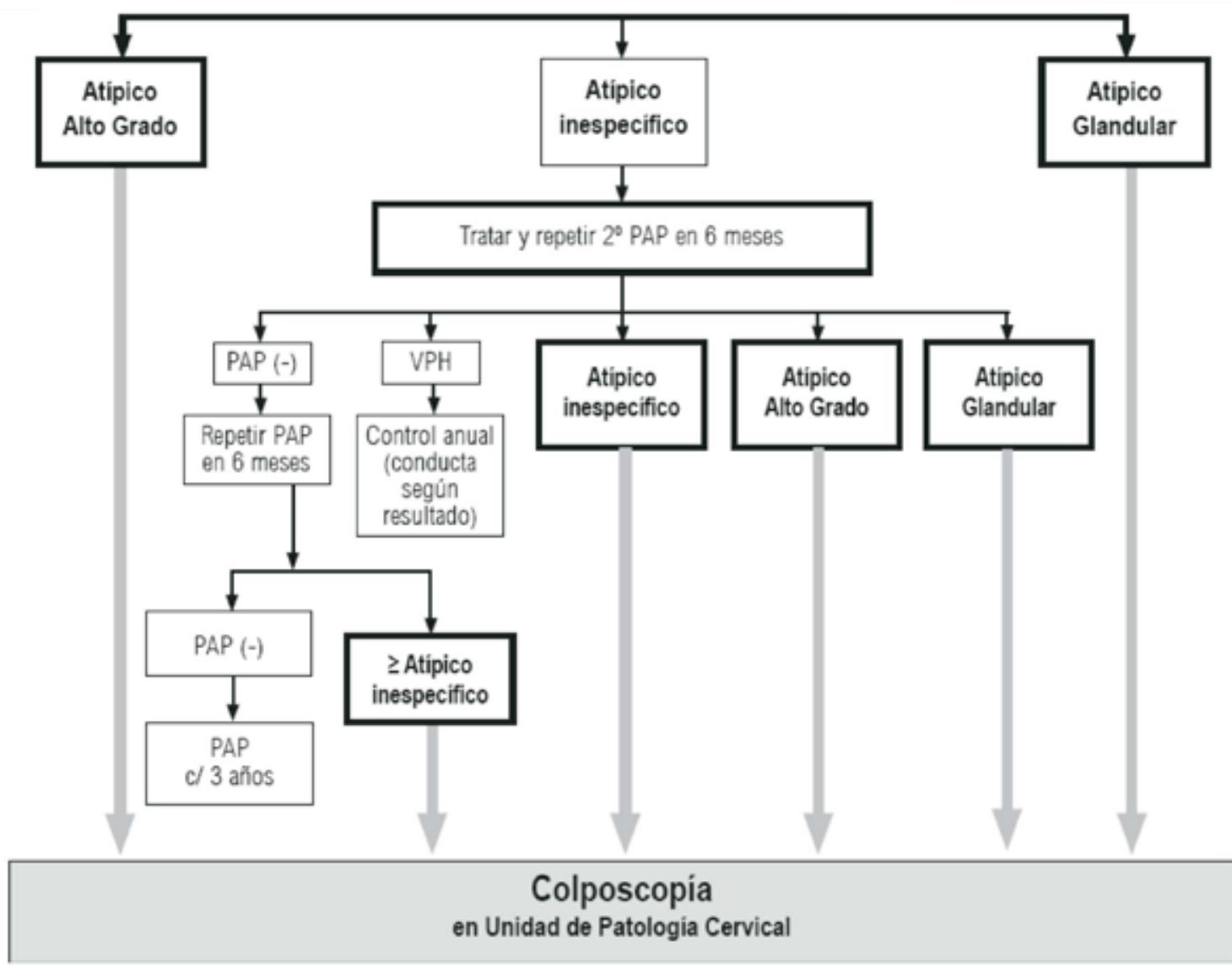

* Adaptación basada en Wright TC, et al. Consensus Guidelines for the Management of Women with Cervical Cytological Abnormalities. JAMA 2002;287(16):2120-9. Ministerio de Salud. Guía Clínica CÁNCER CERVICOUTERINO. Santiago: Minsal, 2010.

Figura 1. Algoritmo de derivación a especialista o Unidad de Patología Cervical del primer PAP atípico según clasificación Behtesda 2001* 


\section{UNIDAD PATOLOGIA CERVICAL O}

EVALUACION ESPECIALISTA

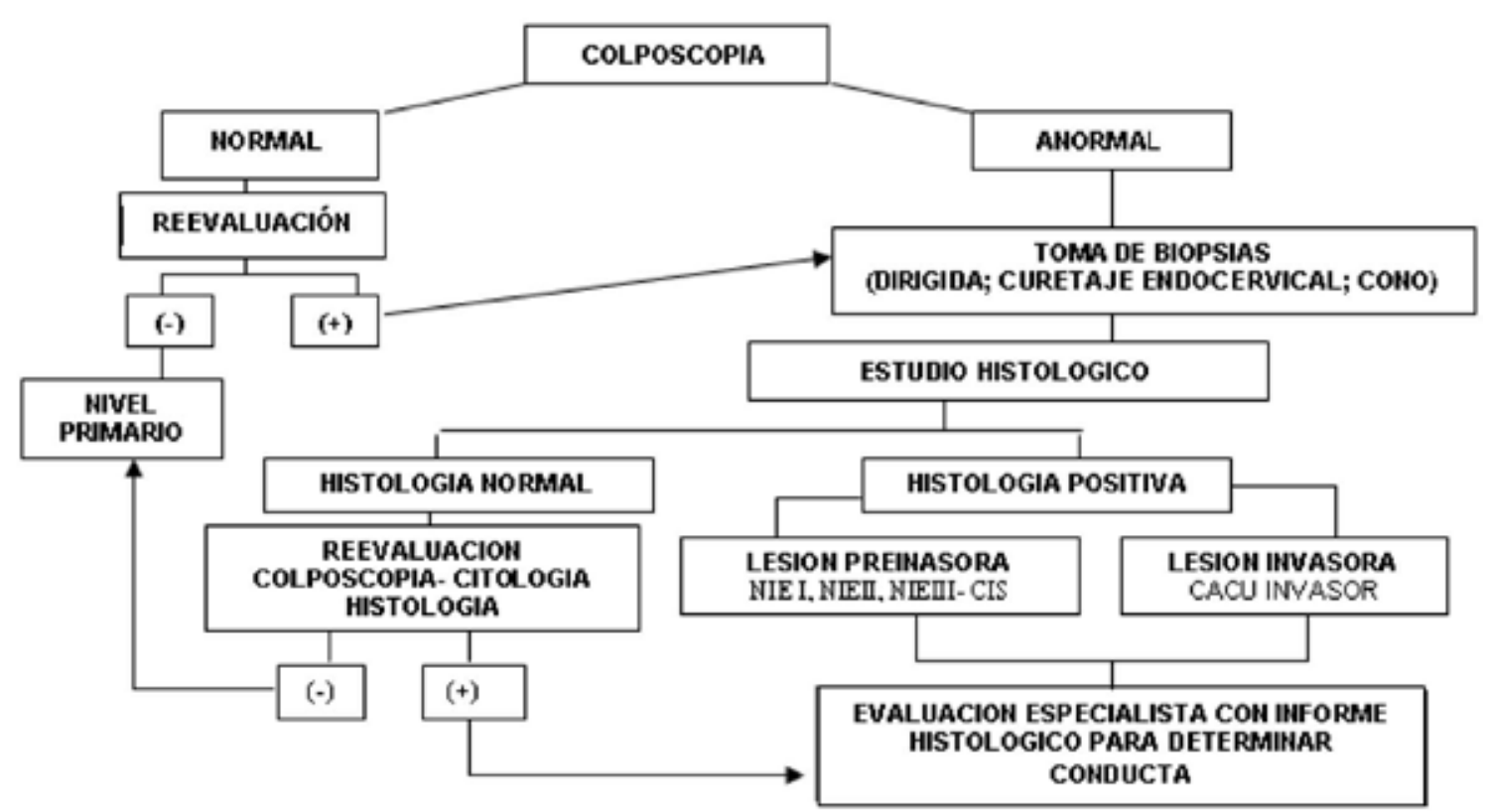

*Ministerio de Salud. Guía Clínica CÁNCER CERVICOUTERINO. Santiago: Minsal, 2010

Figura 2. Algoritmos para confirmación diagnóstica (esquema general).

La segunda versión de esta Guía Clínica en 2010, actualiza y reemplaza a la anterior (49). En Chile, los estudios de seguimiento cito-histológico no han contribuido con evidencia clínica epidemiológica nacional y actualizada a los algoritmos de derivación y confirmación diagnóstica porque no discriminan según tipo de atipias citológicas $(50,51)$.

Por lo anterior, el Ministerio de Salud de Chile determinó fijar como meta la generación de algoritmos clínicos sustentados en evidencias científicas nacionales y adecuadas. Para llevarlo a cabo, se creó la Unidad de Evaluación de Tecnologías Sanitarias que en sus objetivos incluye la identificación de las mejores evidencias científicas para la formulación de Guías Prácticas Clínicas, que son las tecnologías sanitarias que contribuirán al cumplimiento de las metas de impacto del Programa Nacional de Pesquisa y Control del Cáncer Cervicouterino y del Plan Nacional de Salud conducentes al logro de los Objetivos Sanitarios de la década 2011-2020.

\section{CONCLUSIONES}

A la luz de la literatura revisada, por la relevancia que tienen en los índices de morbi-mortalidad por cáncer cérvicouterino, es indiscutible la necesidad de promover estudios epidemiológicos en nuestro medio, sobre la frecuencia y evolución de los resultados de Papanicolau atípicos. Estos estudios generaran evidencia clínica epidemiológica actualizada que permitirá, por una parte, validar o adecuar los algoritmos nacionales de derivación y confirmación diagnóstica, como soporte para la próxima versión programada de la Guía Práctica Clínica de Cáncer Cervicouterino y, por otra, orientar las estrategias de capacitación de los equipos de salud en los diferentes niveles de atención. Es la tarea pendiente del Programa de Pesquisa y Control del Cáncer Cervicouterino de Chile para mejorar su impacto en la salud de la mujer. 
Agradecimientos: Los autores agradecen la asistencia de Bárbara Rivera López en los aspectos relativos al correcto uso del español y al Ministerio de Salud por permitir la publicación de las Figuras originales 1 y 2 .

\section{REFERENCIAS}

1. Wittet S, Tsu V. Cervical cancer prevention and the Millennium Development Goals. Bull World Health Org 2008;86:488-91.

2. Departamento de Estadísticas e Información de Salud. Estadísticas Vitales[Internet]. Chile: Ministerio de Salud; 2011 [consulta el 1 julio 2011] Disponible en: http://deis.minsal.cl/index.asp.

3. Unidad de Cáncer. Programa Nacional de Cáncer Cérvico Uterino. [Internet]. Chile: Ministerio de Salud; 2006 [consulta el 1 julio 2011] Disponible en: http:// www.redsalud.gov.cl/archivos/cancer/Cacu_publico_2006.pdf

4. Howell LP, Wilton M, Bishop J, Afify A. Living With Uncertainty: Equivocal Pap Test Results and the Evolution of ASC Terminology. Diagn Cytopathol 2010;38:221-32.

5. Lacruz C. Nomenclatura de las lesiones cervicales (de Papanicolau a Bethesda 2001). Rev Esp Patol 2003;36:5-10.

6. Pedrosa M, Mattos I, Koifman R, Silva R, Ataide M. Atipias escamosas de significado indeterminado: uma revisão da literatura. DST - J Bras Doenças Sex Transm 2003;15:46-51.

7. National Cancer Institute Workshop. The 1988 Bethesda System for reporting cervical/vaginal cytological diagnoses. JAMA 1989;262:931-4

8. Kurman RJ, Henson DE, Herbst AL, Noller KL, Schiffman $\mathrm{MH}$. Interim guidelines for management of abnormal cervical cytology. The 1992 National Cancer Institute Workshop. JAMA 1994;271:1866-9.

9. Luff RD. The Bethesda System for reporting cervical/ vaginal cytologic diagnoses: Report of the $1991 \mathrm{Be}-$ thesda Workshop. Ed Am J Clin Pathol 1992; 98:152-4.

10. The Bethesda Committee. The Bethesda System for reporting cervical/vaginal cytologic diagnoses. Acta Cytol 1993; 37:115-24.

11. Solomon D, Davey D, Kurman R, Moriarty A, O'Connor $D$, Prey M, et al. The 2001 Bethesda System: Terminology for the reporting results of cervical cytology. JAMA 2002;287:2114-9.

12. Simsir $A$, loffe $O$, Sun $P$, Elgert $P$, Cangiarella J, Levine $\mathrm{PH}$. Effect of Bethesda 2001 on reporting of atypical squamous cells (ASC) with special emphasis on atypical squamous cells-cannot rule out high grade (ASC-H). Diagn Cytopathol 2006; 34:62-66.

13. Lee SJ, Jung KL, Lee JW, et al. Analyses of atypical squamous cells refined by the 2001 Bethesda System: The distribution and clinical significance of follow-up management. Int J Gynecol Cancer 2006;16:664-9.

14. Schiffman M, Adrianza E. ASCUS-LSIL triage study: Design, methods and characteristics of trial participants. Acta Cytol 2000; 44:726-42.

15. Schiffman M, Solomon D. Findings to date from the
ASCUS-LSIL Triage Study (ALTS). Arch Pathol Lab Med 2003;127:946-9.

16. The ASCUS-LSIL Triage Study (ALTS) Group. Results of a randomized trial on the management of cytology interpretations of atypical squamous cells of undetermined significance. Am J Obstet Gynecol 2003;188:1383-92.

17. Sherman ME, Solomon D, Schiffman M. Qualification of ASCUS: A Comparison of Equivocal LSIL and Equivocal HSIL Cervical Cytology in the ASCUS LSIL Triage Study. Am J Clin Pathol 2001;116:386-94.

18. Srodon M, Dilworth HP, Ronnett BM. Atypical squamous cells, cannot exclude high-grade squamous intraepithelial lesion: diagnostic performance, human papillomavirus testing, and follow-up results. Cancer (Cancer Cytopathology) 2006;108:32-8.

19. Davey DD, Neal MH, Wilbur DC, Colgan TJ, Styer PE, Mody DR. Bethesda 2001 implementation and reporting rates: 2003 practices of participants in the College of American Pathologists Interlaboratory Comparison Program in Cervicovaginal Cytology. Arch Pathol Lab Med 2004;128:1224-9.

20. Cox JT, Schiffman M, Solomon D, for the ASCUSLSIL Triage Study (ALTS) Group. Prospective followup suggests similar risk of subsequent cervical intraepithelial neoplasia grade 2 or 3 among women with cervical intraepithelial neoplasia grade 1 or negative colposcopy and directed biopsy. Am J Obstet Gynecol 2003;188:1406-12.

21. Solomon D, Schiffman M, Tarone R, For the ALTS Group. Comparison of Three Management Strategies for Patients With Atypical Squamous Cells of Undetermined Significance: Baseline Results From a Randomized Trial. J Natl Cancer Inst 2001;93:293-9.

22. Wright TC, Jr, Cox JT, Massad LS, Twiggs LB, Wilkinson EJ, ASCCP-Sponsored Consensus Conference. 2001 Consensus Guidelines for the management of women with cervical cytological abnormalities. JAMA 2002;287:2120-9.

23. Wright TC, Massad LS, Dunton CJ, Spitzer M, Wilkinson EJ, Solomon S. The 2006 consensus guidelines for the management of women with abnormal cervical cancer screening tests. Am J Obstet Gynecol 2007; 197:346-55.

24. Sharpless KE, Schnatz PF, Mandavilli S, Greene JF, Sorosky JI. Dysplasia associated with atypical glandular cells on cervical cytology. Obstet Gynecol 2005;105:494-500.

25. McHale MT, Souther J, Elkas JC, Monk BJ, Harrison TA. Is atypical squamous cells that cannot exclude high-grade squamous intraepithelial lesion clinically significant? J Low Genit Tract Dis 2007;11:86-9.

26. Emerson R, Puzanov A, Brunnemer C, Younger Ch, Cramer $\mathrm{H}$. Long-term follow-up of women with atypical squamous cells of undetermined significance (ASCUS). Diagn Cytopathol 2002; 27(3):153-7.

27. Gupta S, Sodhani P, Lal Chachra K, Singh V, Sehgal, A. Outcome of "atypical squamous cells" in a cervical cytology screening program: implications for follow up in resource limited settings. Diagn Cytopathol 2007;35 :677-80.

28. Mood N, Haratian A. Atypical squamous cells of un- 
determined significance: A cytohistologic study. Acta Medica Iranica 2004;42:295-9.

29. Cheung AN, Szeto EF, Ng KM, Fong KW, Yeung $\mathrm{ACH}$, Tsun OK, et al. Atypical squamous cells of undetermined significance on cervical smear. Follow-up study of an Asian screening population. Cancer (Cancer Cytopathol) 2004;102:74-80.

30. Abdullah LS. Pattern of abnormal Pap smears in developing countries: A report from a large referral hospital in Saudi Arabia using the revised 2001 Bethesda System. Ann Saudi Med 2007;27:268-72.

31. Rossi PG, Ricciardi A, Cohet C, Palazzo F, Furnari G, Valle S, et al. Epidemiology and costs of cervical cancer screening and cervical dysplasia in Italy. BMC Public Health 2009;9:71.

32. Mulay K, Meenakshi S, Patra S, Gowrishankar S. A comparative study of cervical smears in an urban Hospital in India and a population-based screening program in Mauritius. Indian J Path Microb 2009;52:34-7.

33. Limpvanuspong B, Tangjitgamol S, Manusirivithaya S, Khunnarong J Thavaramara T, Leelahakorn S. Prevalence of high grade squamous intraepithelial lesions (HSIL) and invasive cervical cancer in patients with atypical squamous cells of undetermined significance (ASCUS) from cervical Pap smears. Southeast Asian J Trop Med Public Health 2008;39:737-44.

34. Abd El All HS, Refaat A, Dandash K. Prevalence of cervical neoplastic lesions and human papilloma virus infection in Egypt: National cervical cancer screening project. Infect Agent Cancer 2007;2:12.

35. Macêdo A, Jorge S, Antoniazi M, Candido E. Atypical squamous cells of undetermined significance: Analysis of microbiology, cytological criteria and clinical conduct. Tumori 2006;92:213-8.

36. Shelen K. Atípias escamosas de significado indeterminado: novas qualificacoes e importancia na conducta clínica. RBAC 2008;40:121-8.

37. Silveira LMS, Cruz ALN, Faria MS. Atipias cervicais detectadas pela citologia em mulheres atendidas em dois hospitais da rede pública de Sao Luís - MA. RBAC 2008;40:115-9.

38. López F, Quezada O, Barrios T, López E. Follow-up of women with ASC-US in Chile. Diagn Cytopathol 2011;39(4):258-63.

39. Selvaggi SM. Reporting of atypical squamous cells, cannot exclude a high-grade squamous intraepithelial lesion (ASC-H) on cervical samples: Is it significant? Diagn Cytopathol 2003;29:38-41.

40. Duncan L, Jacob SV. Atypical squamous cells, cannot exclude a high-grade squamous intraepithelial lesion: The practice experience of a Hospital-Based reference laboratory with this new Bethesda System diagnostic category. Diagn Cytopathol 2005;32:243-6.

41. Patton AL, Duncan L, Bloom L, Phaneuf G, Zafar N. Atypical Squamous Cells, Cannot Exclude a HighGrade Intraepithelial Lesion and Its Clinical Significance in Postmenopausal, Pregnant, Postpartum, and Contraceptive-use Patients. Cancer (Cancer Cytopathol) 2008;114:481-8.

42. Barreth D, Schepansky A, Capstick V, Johnson G, Steed H, Faught W. Atypical Squamous Cells-Cannot Exclude High-Grade Squamous Intraepithelial Lesion (ASC-H): A Result Not to Be Ignored. J Obstet Gynaecol Can 2006;28:1095-8.

43. Yamamoto LSU, Pereira SMM, Etlinger D, Aguiar LS, Sakai YI, Shirata NK, et al. Frequência de diagnóstico de lesões do colo uterino por faixa etária em mulheres atendidas no Programa de Rastreamento Viva Mulher no período de 2004 a 2008. Rev Inst Adolfo Lutz 2009;68:126-32.

44. Okonda S, Wright C, Michelow P. The status of cervical cytology in Swaziland, Southern Africa: a descriptive study. Cytojournal 2009;6:14.

45. Rossi PG, Chini F, Bisanzi S, Burroni E, Carillo G, Lattanzi $A$, et al. Distribution of high and low risk HPV types by cytological status: a population based study from Italy. Infect Agent Cancer 2011;6:1-8.

46. Veiga FR, Russomano F, Camargo MJ, Sampaio AC, Reis A, Tristao MA. Prevalência das lesões intra-epiteliais de alto grau em pacientes com citologia com diagnóstico persistente de ASCUS. Rev Bras Ginecol Obstet 2006;28:75-80

47. García-Perlaza C, Amaya-Guio J, Naranjo E, Ambrosi N. Prevalencia de lesiones escamosas intraepitelial de cervix en pacientes con atipía escamosa de significado indeterminado (ASCUS), en una institución de complejidad media en Bogota, Colombia. Rev Colom Obstet Ginec 2007;58:124-8.

48. Ministerio de Salud. Guía Clínica Cancer Cervicouterino 2. 1a ed. Santiago: Ministerio de Salud, 2005.

49. Ministerio de Salud. Guía Clínica Cáncer Cervicouterino. 2a ed. Santiago: Ministerio de Salud, 2010.

50. Gaete JL, Fuhrer K, Soto R, Rojas JL. Pap atípico escamoso: ¿Un falso negativo?. Clín y Ciencia 2003;1:13-24.

51. Yazigi R, Rodríguez T, Contreras L, Alcaíno M. El significado clínico de dos papanicolaou atípicos consecutivos. Rev Chil Obstet Ginecol 2005;70:386-90. 